\title{
A Chemical Route to Graphene for Device Applications
}

Scott Gilje ${ }^{\dagger}$, Song Han ${ }^{\ddagger}$, Minsheng Wang ${ }^{\ddagger}$, Kang Wang $^{\ddagger}$, Ric Kaner ${ }^{\dagger}$

${ }^{\dagger}$ Department of Chemistry and Biochemistry, University of California Los Angeles, Los Angeles, California, 90095, ${ }^{\ddagger}$ Department of Electrical Engineering, University of California at Los Angeles, Los Angeles, California, 90095

\section{Graphite oxide synthesis:}

Graphite oxide is synthesized from graphite powder (SP-1 grade 325 mesh, Bay Carbon Inc.) by a modified Hummers method as originally presented by Kovtyukhova, et al. ${ }^{1,2}$ The graphite is first pretreated in order to fully oxidize it to graphite oxide (GO). To accomplish this concentrated $\mathrm{H}_{2} \mathrm{SO}_{4}$ $(50 \mathrm{ml})$ is heated to $90{ }^{\circ} \mathrm{C}$ in a $300 \mathrm{ml}$ beaker with $\mathrm{K}_{2} \mathrm{~S}_{2} \mathrm{O}_{8}(10 \mathrm{~g})$ and with $\mathrm{P}_{2} \mathrm{O}_{5}(10 \mathrm{~g})$ added with stirring until all of the reactants are completely dissolved. The mixture is then cooled to $80{ }^{\circ} \mathrm{C}$. Graphite powder $(12 \mathrm{~g})$ is then added to the $\mathrm{H}_{2} \mathrm{SO}_{4}$ solution resulting in bubbling which subsides within 30 minutes. The mixture is kept at $80{ }^{\circ} \mathrm{C}$ for 4.5 hours using a hotplate after which the heating is stopped and the mixture diluted with $2 \mathrm{~L}$ of DI water and left overnight. The following day the mixture is filtered and washed using a 0.2 micron Nylon Millipore filter to remove all traces of acid. The solid is transferred to a drying dish and allowed to dry in air overnight. For the oxidation step of the synthesis, $\mathrm{H}_{2} \mathrm{SO}_{4}(460 \mathrm{ml})$ is placed into a $2 \mathrm{~L}$ Erlenmeyer flask and chilled to $0{ }^{\circ} \mathrm{C}$ using an ice bath. The pretreated graphite is then added to the acid and stirred. $\mathrm{KMnO}_{4}(60 \mathrm{~g})$ is added slowly and allowed to dissolve with the aid of stirring, while the temperature is closely monitored so as not to allow the mixture to go above $10{ }^{\circ} \mathrm{C}$. This mixture is then allowed to react at $35{ }^{\circ} \mathrm{C}$ for 2 hours after which distilled water $(920 \mathrm{ml})$ is added, initially in $20-30 \mathrm{ml}$ aliquots. Since the addition of the water causes the temperature of the mixture to rise rapidly, water addition is carried out in an ice bath so that the 
temperature does not climb above $50{ }^{\circ} \mathrm{C}$. As more water is added, the mixture becomes less reactive until the final $\sim 700 \mathrm{ml}$ can be poured in with no observable resulting rise in temperature. After adding all of the $920 \mathrm{ml}$ of DI water, the mixture is stirred for 2 hours at which time $2.8 \mathrm{~L}$ of DI water is added. Shortly after the dilution with $2.8 \mathrm{~L}$ of water, $50 \mathrm{ml}$ of $30 \% \mathrm{H}_{2} \mathrm{O}_{2}$ is added to the mixture resulting in a brilliant yellow color along with bubbling. The mixture is allowed to settle for at least a day after which the clear supernatant is decanted. The remaining mixture is centrifuged and washed with a total of $5 \mathrm{~L}$ of $10 \% \mathrm{HCl}$ solution followed by $5 \mathrm{~L}$ of $\mathrm{DI}$ water to remove the acid. The resulting solid is dried in air and diluted to make a $2 \% \mathrm{w} / \mathrm{w}$ dispersion that is put through dialysis for 2 weeks to remove any remaining metal. This $2 \%$ aqueous dispersion is used to prepare all of the subsequent dispersions for deposition.

\section{GO Spray coating and reduction:}

The GO dispersion used for film deposition is prepared by weighing out $0.5 \mathrm{~g}$ of the $2 \%$ bulk dispersion and diluting it with $20 \mathrm{ml}$ of Milli-Q water to create a dispersion with a concentration of $\sim$ $500 \mathrm{mg} / \mathrm{L}$ of GO. This darkly colored, translucent, dispersion is then sonicated in a bath sonicator for 30 min after which the liquid appears as a dark transparent color not unlike cola flavored soda. This bulk dispersion is diluted by a factor of 20 to a concentration of $\sim 25 \mathrm{mg} / \mathrm{L}$ for spray coating. The polygrain GO films were spray deposited onto a $\mathrm{Si} / \mathrm{SiO}_{2}$ substrate with a $\mathrm{SiO}_{2}$ layer $300 \mathrm{~nm}$ in depth. The substrates were pre-patterned with 6-point electrodes and cut into squares measuring about 5 x $5 \mathrm{~mm}$. The spraying was done using a Central Pneumatic airbrush model 47791 for a period of 15 sec onto the substrates which were preheated to $150{ }^{\circ} \mathrm{C}$. After spray coating, the substrates were immediately taken away from the heat and placed in a covered container. By modulating the concentration of the dispersion used for the spray coating, different sheet-coverage densities could be obtained. Figure S1 shows a series of AFM images of GO sheets that have been sprayed onto a $\mathrm{SiO}_{2}$ substrate. Using a 15 second spray duration and a starting dispersion concentration of $2.5 \mathrm{mg} / \mathrm{L}$ Figure S1 (a), followed by 
The reduction of the GO films is carried out using $98 \%$ anhydrous hydrazine (Sigma) vapor generated using a bubbler of dry helium in a closed cell. Flowing helium is passed through the cell for at least $1 \mathrm{~h}$ to flush out any ambient moisture. The temperature of the cell is then raised to $80{ }^{\circ} \mathrm{C}$ and the hydrazine bubbler started at a $110 \mathrm{cc} / \mathrm{min}$ flow rate in order to generate hydrazine vapor. The increased temperature is needed to prevent the condensation of water/hydrazine onto the films as well as to facilitate the reduction of GO. After $2.5 \mathrm{~h}$ the reaction is complete and the hydrazine flow along with the heating stopped. Helium is continually passed over the substrates overnight at a $30 \mathrm{cc} / \mathrm{min}$ flow rate in order to remove any traces of unreacted hydrazine from the films. The total time required for the spray deposition and reduction of the graphite oxide is approximately 4 hours.

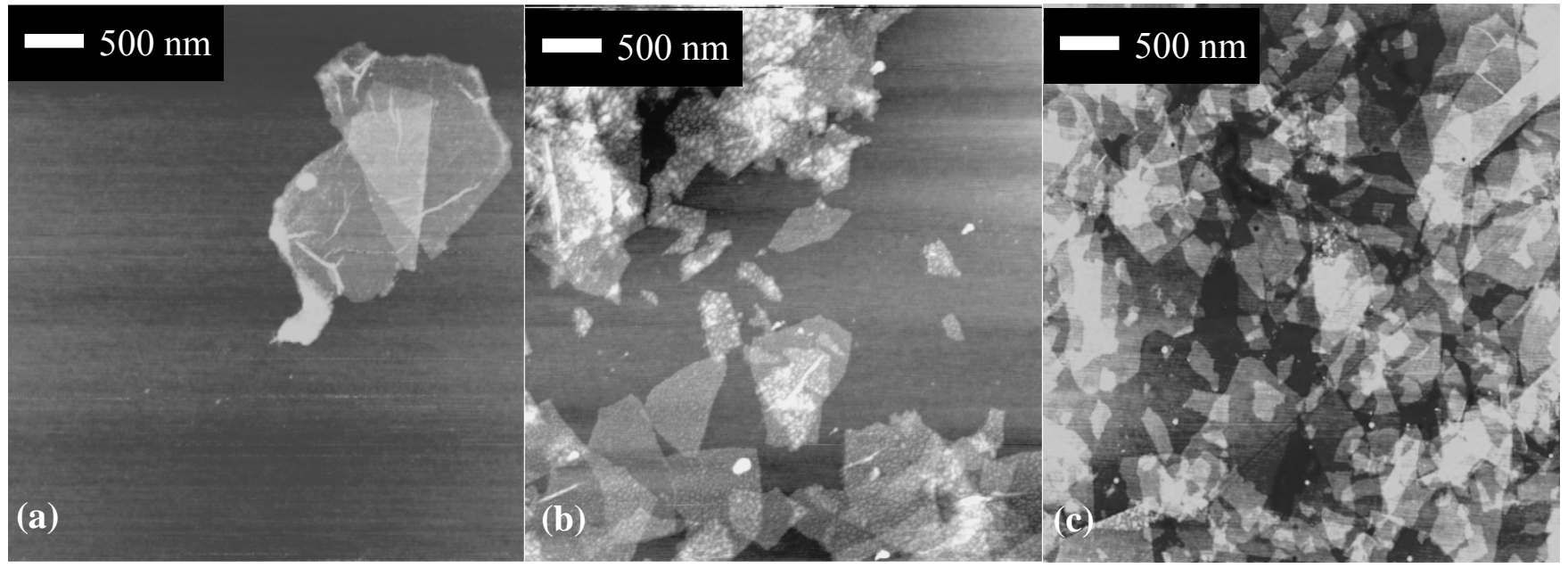

Figure S1 AFM images depicting a series of different GO sheet coverage densities on $\mathrm{SiO}_{2}$, achieved by increasing the concentration of the GO dispersion used during a 15 second spray deposition from (a) $2.5 \mathrm{mg} / \mathrm{L}$ to (b) $12.5 \mathrm{mg} / \mathrm{L}$ to (c) $25 \mathrm{mg} / \mathrm{L}$.

\section{Electrode fabrication and electrical property measurements:}

Source/drain electrodes are fabricated on the graphite oxide and reduced graphite oxide films by electron beam lithography followed by metal deposition of $3 \mathrm{~nm}$ Ti and $30 \mathrm{~nm}$ Au. The channel length is $500 \mathrm{~nm}$ and channel width is $20 \mu \mathrm{m}$, which ensures the existence of graphite oxide films between 
source and drain electrodes.

\section{Characterization:}

X-ray diffraction (XRD) is carried out using a PANalytical XPert Pro diffractometer with $\mathrm{Cu} \mathrm{K} \alpha$ radiation $(\lambda=1.5418 \AA$ ). Before taking $\mathrm{X}$-ray scans the GO is dried for $48 \mathrm{~h}$ under vacuum at room temperature followed by $24 \mathrm{~h}$ under vacuum over $\mathrm{P}_{2} \mathrm{O}_{5}$ which acts as a drying agent. ${ }^{1}$ Powder X-ray diffraction can be used to verify that the oxidation reaction has reached completion since the introduction of oxygen moieties expands the interplanar galleries in graphite from $3.34 \AA$ to $\sim 6.9 \AA$. ${ }^{1,3,4}$ Our graphite oxide exhibits a characteristic peak at 12.75 degrees $2 \theta$ corresponding to the 002 interplanar spacing of $6.94 \AA$, while the most intense peak from the starting graphite at 26.4 degrees $2 \theta$, corresponding to a d-spacing of $3.34 \AA$, is completely absent. The d-spacing of slightly $>6.9 \AA$ for the synthesized GO indicates that despite drying under vacuum for $24 \mathrm{~h}$, some water has been absorbed by the GO.

Thermogravimetric analysis (TGA) is carried out using a Perkin Elmer Pyris Diamond TG/DTA. The GO is dried prior to taking thermograms using the same method as that used before taking XRD scans. For TGA, the temperature was ramped from $25-800^{\circ} \mathrm{C}$ at a rate of $5{ }^{\circ} \mathrm{C} / \mathrm{min}$ under dry air. TGA data is consistent with the formation of graphite oxide (GO) containing some residual water. A TGA indicates weight loss at $\sim 200{ }^{\circ} \mathrm{C}$ and $\sim 450{ }^{\circ} \mathrm{C}$ corresponding to the loss of water and decomposition of GO, respectively. ${ }^{5}$ The GO is completely decomposed by the time the temperature reaches $600{ }^{\circ} \mathrm{C}$. This is in sharp contrast to the parent graphite which is stable in air to slightly above $600{ }^{\circ} \mathrm{C}$.

Elemental analysis was performed by Desert Analytics, Tucson, AZ. Raman spectra were taken using a Jobin Yvon HR800 with a He-Ne Laser at $632.8 \mathrm{~nm}$ excitation and a $300 \mathrm{~cm}^{-1} \pm 1 \mathrm{~cm}^{-1}$ holographic grating. Elemental analysis of the GO product indicates that its atomic composition (wt\%) is: C, 50.15 \%; O, $38.16 \% ; \mathrm{H}, 1.03 \%$; N, $0.4 \%$; Residual Ash, $9.2 \%$;. This yields a C:O:H ratio of: 4:2.3:1 with a $\mathrm{C}: \mathrm{O}$ ratio of 1.8 indicating that our GO material is highly oxidized, but well within the range of reported 
values for GO prepared from natural graphite. ${ }^{6,7}$ The accepted nominal formula for GO is given as $\mathrm{C}_{8} \mathrm{O}_{2}(\mathrm{OH})_{2}$ which translates into an atomic composition (wt \%) of: $\mathrm{C}, 59.25 \% ; \mathrm{O}, 39.5 \%$; $1.25 \%$ yielding a $\mathrm{C}: \mathrm{H}: \mathrm{O}$ of ratio $4: 2: 1{ }^{1}$ More important than the actual weight percentage, may be the theoretical atomic $\mathrm{C}: \mathrm{O}$ ratio of 2.0 , which is generally accepted as the point at which graphite is completely converted to GO. In most cases, this ratio is non-ideal and has a reported range from 1.8 to 2.5. ${ }^{1,8}$ In the case of GO synthesized from natural graphite, ratios as low as 1.3 have also been reported. ${ }^{1,3,4}$ Based on this data, our GO material appears to be highly oxidized and most likely contains some absorbed water along with residual phosphorus and potassium from the reaction despite repeated washing. These contaminants are likely residue from the pre-oxidation step and do not interfere with the deposition.

Transmission electron microscopy (TEM) analysis was carried out on a JEOL 100CX TEM using a formvar/carbon 300 mesh copper grid. The samples were cast onto the TEM grid from an aqueous dispersion at a concentration of $25 \mathrm{mg} / \mathrm{L}$.

Atomic force microscopy (AFM) was carried out using a Digital Instruments Multimode ${ }^{\mathrm{TM}}$ SPM equipped with an AS-12 ("E") scanner operated in tapping mode in conjunction with a V-shaped tapping tip (Applied Nanostructures SPM model: ACTA). The images were taken at a scan rate of 2 Hz. Optical images were taken using an Olympus BX40 microscope.

Raman results were taken by RENISHAW inVia Raman Microscope with laser power at $10 \mathrm{~mW}$ at wave length of $532 \mathrm{~nm}$.

\section{REFERENCES:}

(1) Kovtyukhova, N. I.; Ollivier, P. J.; Martin, B. R.; Mallouk, T. E.; Chizhik, S. A.; Buzaneva, E. V.; Gorchinskiy, A. D.; Chem. Mater.1999, 11(3), 771-778.

(2) Hummers, W. S., Jr.; Offeman, R. E.; J. Am. Chem. Soc. 1958, 80, 1339. 
(3) Scholz, W.; Boehm, H. P.; Zeitschrift fuer Anorganische und Allgemeine Chemie. 1969, 369(3-6), 327-340.

(4) Nakajima, T.; Mabuchi, A.; Hagiwara, R.; Carbon. 1988, 26(3), 357-361.

(5) Bissessur, R.; Liu, Peter K. Y.; White, W.; Scully, S. F.; Langmuir 2006, 22(4), 1729-1734.

(6) Kyotani, T.; Suzuki, K.; Yamashita, H.; Tomita, A.; Tanso 1993, 160, 255-265.

(7) Slabaugh, W. H.; Seiler, B. C.; J. of Phys. Chem.1962, 66, 396-401.

(8) Bourlinos, A. B.; Gournis, D.; Petridis, D.; Szabo, T.; Szeri, A.; Dékány, I.; Langmuir 2003, 19(15), 6050-6055. 